\title{
Improved Traction for a Mobile Robot Traveling on the Heart
}

\author{
N.A. Patronik ${ }^{1}$, T. Ota ${ }^{2}$, M.A. Zenati ${ }^{1,2}$, and C.N. Riviere ${ }^{1}$ \\ ${ }^{1}$ The Robotics Institute, Carnegie Mellon University, Pittsburgh, PA, USA \\ 2 Division of Cardiothoracic Surgery, University of Pittsburgh, Pittsburgh, PA, USA
}

\begin{abstract}
This document describes the effects of several design parameters on the traction generated by the suction pads of a mobile robot that walks on the surface of the heart. HeartLander is a miniature mobile robot that adheres to the epicardial surface of the heart using suction, and can travel to any desired location on the heart to administer therapeutic applications. To maximize the effectiveness of locomotion, the gripper pads must provide sufficient traction to avoid slipping. Our testing setup measured the force applied to the gripper pad adhering to ovine epicardial tissue, and recorded overhead video for tracking of the pad and tissue during an extension. By synchronizing the force and video data, we were able to determine the point at which the pad lost traction and slipped during the extension. Of the pads tested, the pad with no suction grate achieved maximum traction. Increasing the extension speed up to $20 \mathrm{~mm} / \mathrm{s}$ resulted in a corresponding increase in traction. Increasing the vacuum pressure also improved the traction, but the magnitude of the effect was less than the improvement gained from increasing extension speed.
\end{abstract}

\section{INTRODUCTION}

$\mathrm{M}^{2}$ any minimally invasive cardiac surgeries are currently being performed with assistance from robotic tools to compensate for the diminished dexterity and visualization that result from the lack of direct access to the operative site [1]. Although robotic teleoperated systems provide improved working conditions to the surgeon, they typically require lung deflation and mechanical stabilization, and have access limitations for cardiac surgery [2]. HeartLander is a mobile robot that overcomes these limitations for intrapericardial therapies [3]. By adhering to the epicardial surface of the heart using vacuum pressure, mechanical stabilization is obviated because the robot is in the frame of reference of the beating heart. Meanwhile, the locomotive capabilities of HeartLander eliminate the need for lung deflation and provide improved operational access.

The locomotion modality of HeartLander is similar to that of an inchworm or leech. In the first half of the cycle, it grips the heart surface with its rear pad to provide the reaction force necessary for extending the front body section forward. In the second half of the cycle it grips the tissue with the extended front pad, then releases and retracts the rear pad. The distance between the body sections is controlled by a set of motors through a wire-actuated

Manuscript received April 24, 2006. This work was supported by the National Science Foundation (grant no. EEC-9731748) and a NASA GSRP Fellowship (NNG05GL63H). mechanical transmission. Each locomotion cycle generates a forward (or backward) step equal to the extension distance between the two body sections. The efficiency of locomotion, however, is dependent on the traction generated by the gripper pads on the body sections. HeartLander operates within the intrapericardial space between the epicardial surface of the heart and surrounding pericardial sac. Naturally, this space exists only as a thin layer of lubricating fluid, and thus HeartLander must create its own space during locomotion by deforming the epicardium and pericardium. Figure 1 shows HeartLander walking in the anterior intrapericardial space of a beating porcine heart in vivo during recent testing [4]. Although locomotion was successfully demonstrated, loss of traction during the extension and retraction phases was noticeable. This causes the efficiency of locomotion to decrease, and may make locomotion around the posterior portion of the heart impossible due to the additional compression from the surrounding organs. For this reason, it is critical that the gripper pads be designed to maximize traction when vacuum pressure is applied to the tissue.

Although there has been research into the traction of mobile robots in the human body by Rentschler et al., their analysis was for a wheeled vehicle and is thus difficult to adapt here [5]. Additionally, research into grouser sizes and patterns from the areas of terramechanics and field robotics

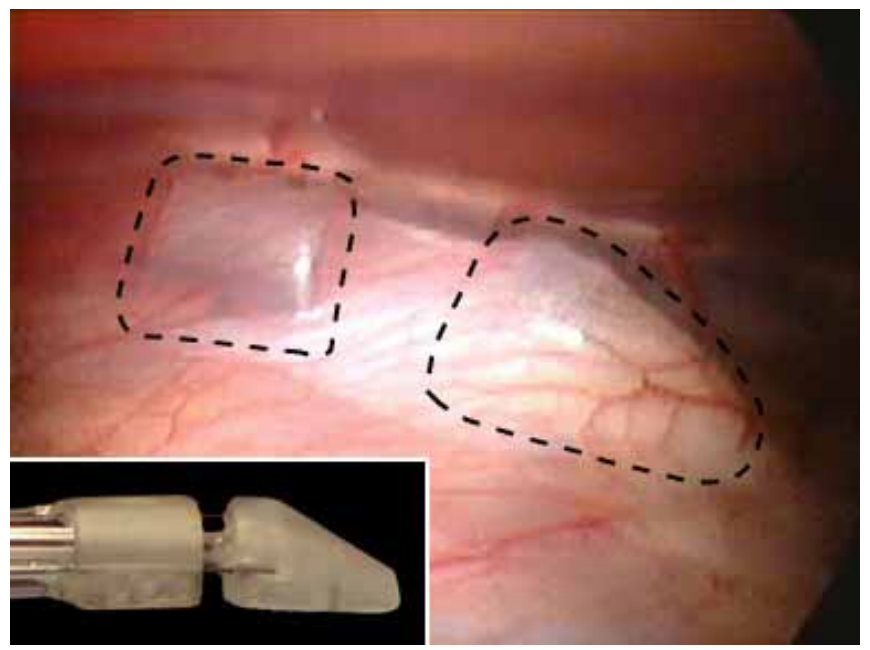

Fig. 1. Locomotion of HeartLander on the epicardial surface of a beating porcine heart with chest closed. The body sections (highlighted) of the robot are visibly distending the pericardium. Video was recorded by an intercostal scope. Inset shows the profile of the robot for clarity. 
is difficult to apply to the domain of medical robotics due to the vast differences in the mechanical structures of soil and biological tissue [6],[7]. For these reasons, we have evaluated the tractive performance of eleven different gripper pads over a range of extension speeds and vacuum pressures.

\section{METHODS}

\section{A. Traction Considerations}

The traction force $\left(F_{f}\right)$ generated by a gripper pad is a function of the vacuum pressure $\left(P_{v}\right)$, the area of the tissue exposed to the vacuum $\left(A_{v}\right)$, and the effective coefficient of friction of the pad when the tissue is engaged $(\mu)$. These factors are related by the following equation:

$$
F_{f}=P_{v} A_{v} \mu \text {. }
$$

The maximum vacuum pressure applied to the epicardium must remain below the value at which permanent damage is done to the tissue. The typical vacuum pressure applied to the epicardium for mechanical stabilization during beatingheart surgery is $400 \mathrm{mmHg}$, although it can be temporarily increased up to $600 \mathrm{mmHg}$ without adverse effects [8],[9]. The area of the tissue exposed to the vacuum pressure is limited by the footprint of the body sections of the robot, which must remain small relative to the curvature of the heart to achieve an effective seal with a stiff pad. Because the vacuum pressure and the area of gripped tissue are thus limited, we must attempt to maximize the effective coefficient of friction of the pad in order to maximize the traction force.

\section{B. Gripper Pad Designs}

Eleven gripper pads were designed to explore the effects of several design parameters on the effective coefficient of friction (Fig. 2). The first eight gripper pads draw the epicardial tissue against a grate with a pattern of perforations that expose the tissue to the vacuum pressure. The use of a grate ensures that large biological particles are not drawn into the vacuum chamber and clog the vacuum supply line from the pump. Although using a grate reduces the area of the tissue exposed to the vacuum pressure, small projections between the perforations can be used to enhance the grip of the tissue. The use of such tissue engaging features has been used in commercially available cardiac stabilizers, as well as in mobile robotics [5],[7],[10]. Pad 1 had a flat grate with no projections, while Pads 2 through 8 featured projections with varying profiles. Pads 7 and 8 also featured serrations around the perimeter of the pad bottom. Pad 9 employed a different style grate that was more similar to the tread pattern on a wheeled vehicle. Pads 10 and 11 did not have suction grates at all. Although this open design may prove more susceptible to clogging, it maximizes the area of tissue exposed to the vacuum source. Pad 10 featured projections on the ceiling of the suction chamber, while Pad 11 did not.
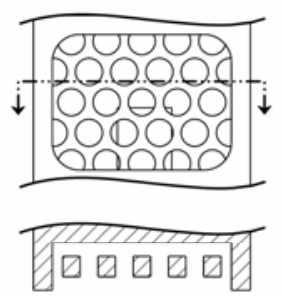

Pad 1: Grate with no teeth

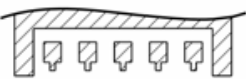

Pad 4: Grate with triangular teeth

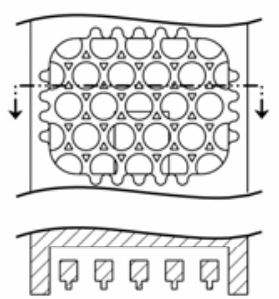

Pad 7: Grate with triangular teeth

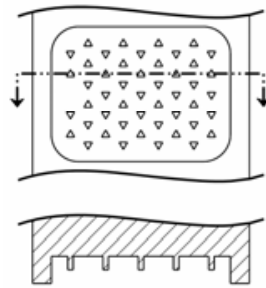

Pad 10: Open with triangular teeth

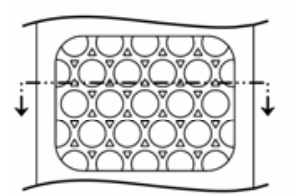

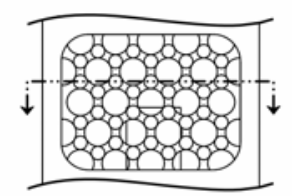

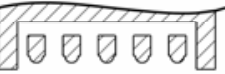

Pad 2: Grate with spherical teeth
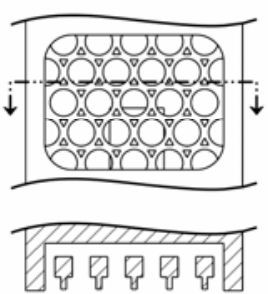

Pad 5: Grate with longer tri. teeth

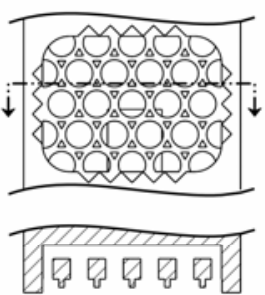

Pad 8: Grate with triangular teeth

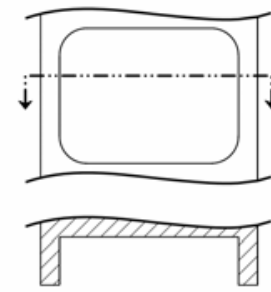

Pad 11: Open chamber
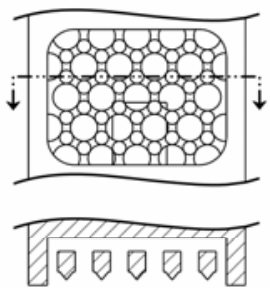

Pad 3: Grate with conical teeth
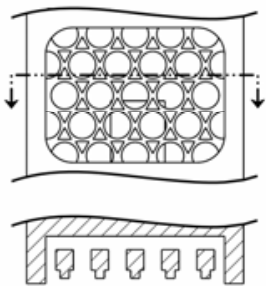

Pad 6: Grate with larger tri. teeth

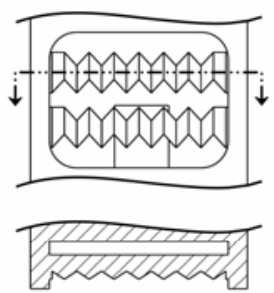

Pad 9: Treads

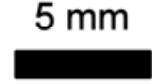

Fig. 2. Bottom surfaces and profile cross sections for the gripper pads.

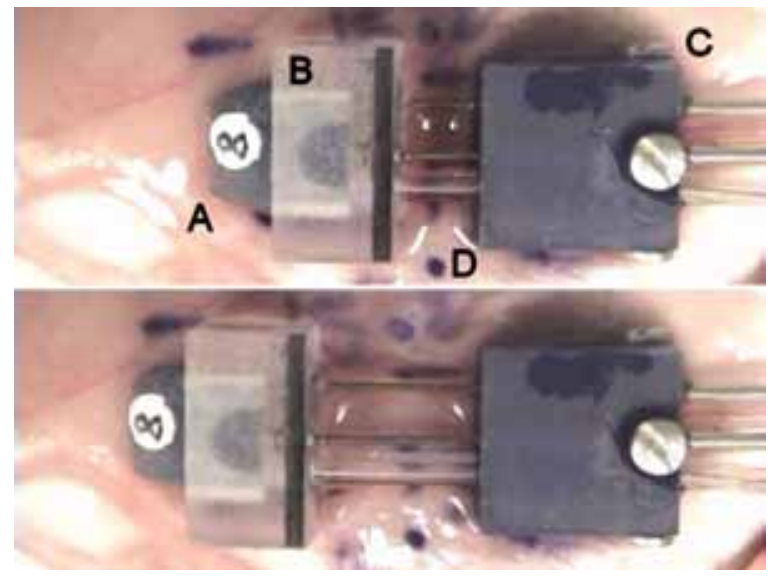

Fig. 3. View of the testing setup from the video recorded during a trial. Shown are the (A) gripper pad, (B) gripper pad holder, (C) rear, stationary pad, and (D) tissue ink mark. It can be seen that the tissue ink mark moves up and left as the tissue between the front and rear grippers is stretched. 


\section{Traction Testing Apparatus}

The traction of each gripper pad was evaluated during vacuum prehension of an excised ovine (sheep) heart by applying a shear force sufficient to cause the pad to slip over the heart. The maximum traction force occurred just prior to slipping. The gripper pad was driven forward at a constant speed over a fixed distance by a stepper motor through a wire-actuated transmission (Fig. 3). The reaction force for the gripper pad being tested was provided by the rear suction pad, which was rigidly fixed to the epicardial surface due to its larger size and higher vacuum pressure. The gripper vacuum pressure, extension speed, and extension distance were all controlled through a computer interface. A load cell in the mechanical transmission measured the force applied to the front gripper pad by the motor. A video camera located orthogonally overhead recorded each extension trial so that the positions of the front gripper pad and an ink mark on the epicardium could be tracked using traditional computer vision methods.

All gripper pads were subjected to a $10-\mathrm{mm}$ extension at a speed of $2 \mathrm{~mm} / \mathrm{s}$, and vacuum pressure of $400 \mathrm{mmHg}$. The gripper pad with the highest maximum traction force (i.e. force at the time of slip) was then tested over a range of extension speeds and vacuum pressures to determine the effects of varying these parameters. All tests were performed in the same location on the epicardial surface of the left ventricle within 4 hours of the heart being excised. The heart was kept moist using a physiological saline solution.

\section{RESULTS}

There were several distinct phases in the traction force measurement of each extension trial, which were typical given the stress-strain properties for muscle tissue (Fig. 4) [11]. Following the initial rise as the motor overcame the friction of the transmission, the force rose exponentially for the first 3-mm of the extension as the epicardial tissue was stretched over its normal physiological range. The force then became linear as the tissue was stretched past its normal operating range from 3 to $5 \mathrm{~mm}$. Lastly, the traction force reached a maximum and became roughly constant as the gripper pad lost traction and began to slip over the epicardial tissue. This slip transition occurred when the force required to further stretch the epicardial tissue exceeded the maximum traction force that could be generated by the gripper pad. By tracking the position of the ink mark on the epicardial tissue, the point at which the pad lost traction was also independently estimated as the time at which the ink mark stopped moving. For our testing, these independent measures of the location of the slip transition closely agreed for all trials, as can be seen in Figure 4.

In order to explore the possible effects of cycle dependent factors that influence the stress-strain relationship of the epicardial tissue, e.g. preconditioning, we tested three different pads in the same location on the tissue for two trials each. The average difference in extension distance at the time of slip for the three pads was $0.15 \mathrm{~mm}$, with a maximum difference of $0.21 \mathrm{~mm}$. The average difference in force measurement at the slip point was $0.08 \mathrm{~N}$, with a maximum difference of $0.14 \mathrm{~N}$. This close agreement in both extension distance and force value at the slip points for the three pads illustrated that the tissue did not experience a significant change in stress-strain properties over multiple trials.

The results of the trials in which the gripper pad was varied for a $10-\mathrm{mm}$ extension at a speed of $2 \mathrm{~mm} / \mathrm{s}$ and pressure of $400 \mathrm{mmHg}$ are shown in Figure 5. It can be seen that the pads generated maximum traction forces that ranged from 2.7 to $4.0 \mathrm{~N}$. The pad without a perforated grate or projections ( $\mathrm{Pad} 11$ ) generated the highest traction force before slipping at $4.0 \mathrm{~N}$.

The effect of varying the speed for Pad 11 was then investigated for speeds ranging from 2 to $30 \mathrm{~mm} / \mathrm{s}$. The maximum traction force increased with increasing speed up to $20 \mathrm{~mm} / \mathrm{s}$, then failed to increase further with increasing speed (Fig. 6).

Lastly we investigated the effect of increasing the vacuum pressure from 400 to $600 \mathrm{mmHg}$ for Pad 11 over two speeds (5 and $30 \mathrm{~mm} / \mathrm{s}$ ). Figure 7 shows that for both speeds the maximum traction force increased linearly with increasing vacuum pressure.

\section{DISCUSSION}

This set of experiments has revealed specifications for the gripper pad design, extension speed, and vacuum pressure that yield the maximum traction on ovine epicardium.

The gripper pad without a perforated grate (Pad 11) generated the highest traction force $(4.0 \mathrm{~N})$ of the set of gripper pads tested with a fixed extension speed of $2 \mathrm{~mm} / \mathrm{s}$ and vacuum pressure of $400 \mathrm{mmHg}$. This is most likely due to the fact that Pad 11 exposed the largest area of the epicardial tissue to the vacuum pressure, and therefore

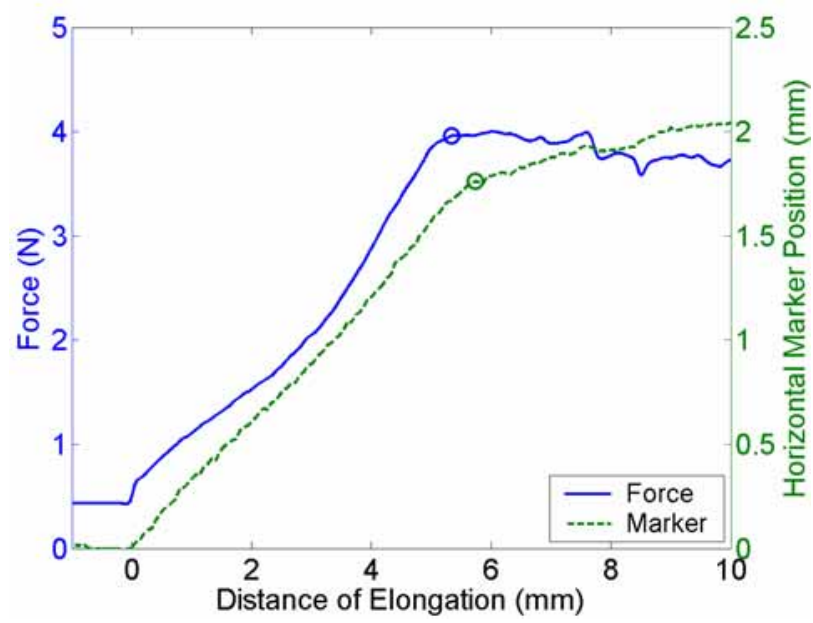

Fig. 4. The force recorded during an extension trial with Pad 11 (solid line), and the corresponding horizontal displacement of an ink mark on the epicardial tissue (broken line) from the same extension. The circles show the slip estimates from each independent measurement. 


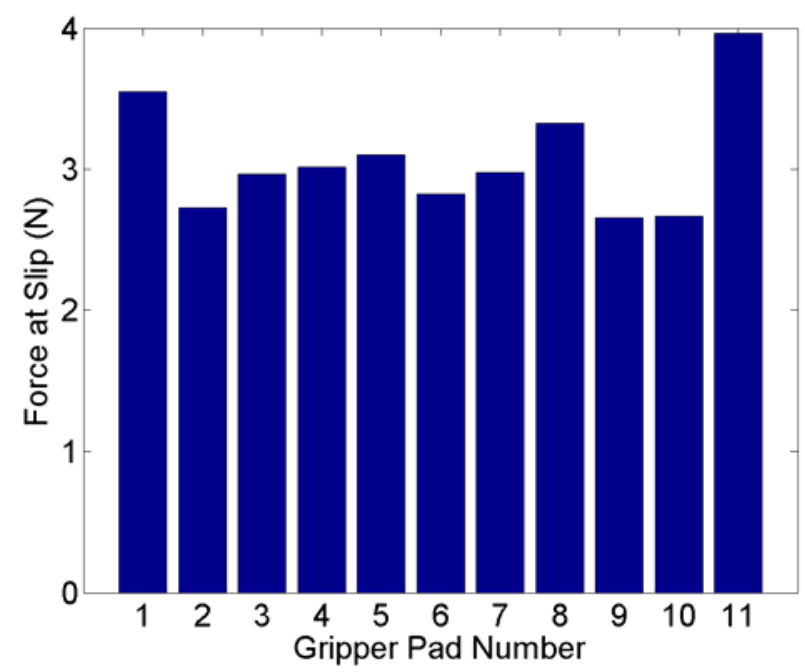

Fig. 5. The forces recorded at the loss of traction for the different gripper pads extended at a speed of $2 \mathrm{~mm} / \mathrm{s}$ with vacuum pressure of $400 \mathrm{mmHg}$.

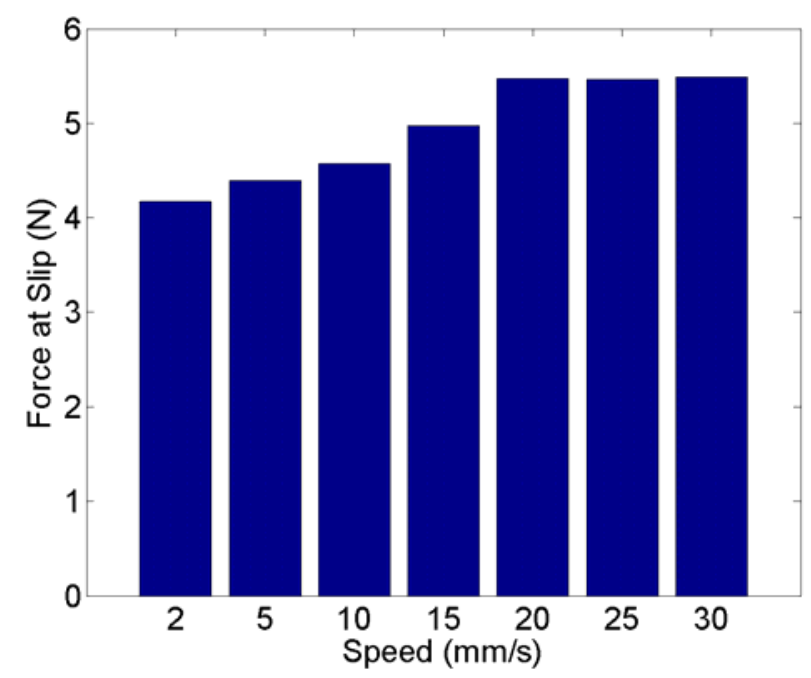

Fig. 6. The forces recorded at the loss of traction over a range of extension speeds with gripper Pad 11 and vacuum pressure of $400 \mathrm{mmHg}$.

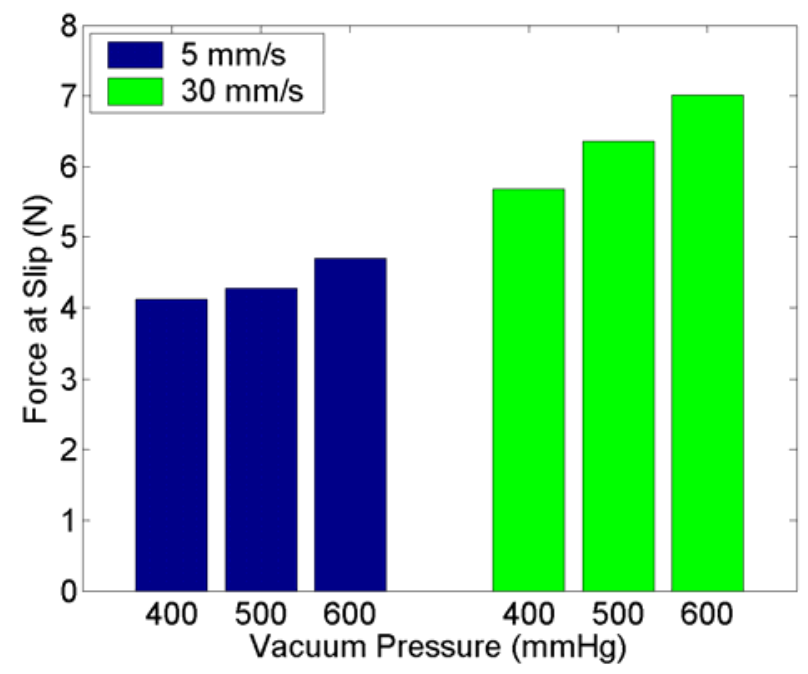

Fig. 7. The forces recorded at loss of traction over a range of vacuum pressures with gripper Pad 11 and two different extension speeds. generated the highest normal force of all the gripper pads. For example, the tissue area exposed to vacuum was $32 \mathrm{~mm}^{2}$ for Pad 11, but was only $15 \mathrm{~mm}^{2}$ for any of the pads with the suction grate. An increase in the maximum traction force directly proportional to the increase in the exposed tissue area was not observed, most likely due to the edge effects of the tissue engaging the perforations of the pads with grates. The depth to which the tissue became engaged was also the highest for Pad 11, which may have improved traction.

For Pad 11 the optimal extension speed was $20 \mathrm{~mm} / \mathrm{s}$, as increasing the speed past this value failed to yield a corresponding increase in the maximum traction force. The viscoelasticity of epicardial tissue most likely caused an increase in the tissue stiffness with the increasing speed, thus leading to higher reaction forces and traction [11].

Although increasing the vacuum pressure resulted in a linear increase in the traction, it is clear from Figure 7 that a more significant improvement in traction resulted from increasing the extension speed. On a diseased heart it may prove undesirable to increase the vacuum pressure above $400 \mathrm{mmHg}$, which will be explored in future research.

The design parameters described above will be implemented in the HeartLander system and tested on a beating porcine heart with a closed chest. By improving the traction of the gripper pads during locomotion, we will improve efficiency and expand our access within the intrapericardial space.

\section{REFERENCES}

[1] Diodato MD, Damiano RJ. 2003. Robotic cardiac surgery: overview. Surg Clin North Am, 83(6): 1351-1367.

[2] H. Shennib, M.J. Mack, A.G.Lee. "A Survey on Minimally Invasive Coronary Artery Bypass Grafting,” Ann Thorac Surg. 1997; 64:110115.

[3] N.A. Patronik, M.A. Zenati, C.N. Riviere. "Preliminary evaluation of a tethered robotic device for navigation on the beating heart," Computer Aided Surgery. (2005); in press, Invited Paper.

[4] T. Ota, N. A. Patronik, C. N. Riviere, M. A. Zenati. "Percutaneous access to the epicardium using a miniature crawling robotic device," The Annual Meeting of the International Society of Minimally Invasive Cardiac Surgery, submitted for publication.

[5] Rentschler, M., Dumpert, J., Platt, S., Iagnemma, K., Oleynikov, D., Farritor, S., "Modeling, Analysis, and Experimental Study of In Vivo Wheeled Robotic Mobility", IEEE Transactions on Robotics, in press.

[6] R. N. Yong, A. F. Youssef and H. El-Mamlouk. "Soil deformation and slip relative to grouser shape and spacing”, Journal of Terramechanics. 1978; 15 (3): 129-144.

[7] Bauer, R.; Leung, W.; Barfoot, T., "Experimental and simulation results of wheel-soil interaction for planetary rovers," International Conference on Intelligent Robots and Systems, 2005. (IROS 2005). pp 586- 591.

[8] Borst C, Jansen EW, Tulleken CA, Grundeman PF, Mansvelt Beck HJ, van Dongen JW, Hodde KC, Bredee JJ. Coronary artery bypass grafting without cardiopulmonary bypass and without interruption of native coronary flow using a novel anastomosis site restraining device ("Octopus"). J Am Coll Cardiol. 1996 May;27(6):1356-64.

[9] Jansen EW, Lahpor JR, Borst C, Grundeman PF, Bredee JJ.Off-pump coronary bypass grafting: how to use the Octopus Tissue Stabilizer. Ann Thorac Surg. 1998 Aug;66(2):576-9.

[10] H.L. Green and J.K. Wallen, "Tissue stabilizer and methods of use," US Patent No. US 2003/0088150 A1. 2003.

[11] Y.C. Fung. Biomechanics: Mechanical Properties of Living Tissues. Springer. 1993. 